$<$ Original Article $>$

\title{
Conservative neural symmetry of the caprine mandible
}

\author{
Pere M. Parés-Casanova* \\ Department of Animal Production, University of Lleida, 25198 Lleida, Spain \\ (Received: April 16, 2013; Revised: July 7, 2013; Accepted: August 26, 2013)
}

\begin{abstract}
Fifteen dry dentate and complete mandible samples from the White Rasquera goat breed were studied for symmetry. Thirty-one landmarks were digitally located on the images of the lateral and medial aspects of each hemimandible. Distances between these landmarks allowed the evaluation of the whole hemimandible and also the neural mandible. In the studied samples, the mandible was rather symmetrical, especially in the medial neural part, and in general, there was no side dominance. Only the diastema differed significantly between the sides, and this was related to the rostral part (incisive arch). The incisive region was the least symmetrical region of the caprine mandible, indicating a modular structure more conservative for the neural part. If unsigned asymmetry is interpreted as a measure of developmental stability, then the studied breed presented a marked ability to develop in good fitness despite the harsh environment. The measurements presented here can also be used as a reference for researchers designing experimental studies, especially on mandibular catch-up growth, and as an aid for zooarchaeologists comparing results from dead animals with those from living goat populations.
\end{abstract}

Keywords : jaw, mandibular foramen, mandibular nerve, neural symmetry, White Rasquera goat

\section{Introduction}

The mandible has a complex origin involving both endochondral and intramembranous developments. Its ossification takes place in the membrane covering the outer surface of the first branchial arch [6] from different ossification centres [1]. The mandible appears in adults as a single bone. However, various portions or subunits are found during its development [11]. The main ossification centres correspond to the incisive part, the ventral margin of the molar part, the alveolar margin, the condylar process and the coronoid process [1]. Hence, developmental processes are expected to be different for each centre. Development of the alveolar margin is especially complex as it converts the mandibular nerve's traject into a canal [1]. The bone surrounding this mandibular nerve can be seen as the primitive corpus of the mandible or the neural part of the mandible and forms one unit to which the other parts are attached [2].

In this paper, we present the first analysis of symmetry based on variations in the hemimandibles of a domestic goat breed. We hypothesised that the neural part of the mandible was bilaterally more symmetrical than the rest. The body of the mandible contains the mandibular canal, through which the mandibular artery and vein and the mandibular alveolar nerve pass [6]. The mandibular canal has its caudal opening in the mandibular foramen on the medial surface of the mandible; it passes rostrally, ventral to the dental alveoli and ends in the mental foramen on the lateral surface of the interalveolar margin [6]. This whole part was considered the neural mandible in this research. The parameters assessed in this study involved the foramina of the mandibular canal to characterise the neural mandible and compare it to the size of the mandible and its processes. The measurements presented here can also be used as a reference for researchers designing experimental studies, especially on mandibular catch-up growth, and as an aid for zooarchaeologists comparing results from dead animals with those from living goat populations.

\section{Material and Methods}

Fifteen dry dentate and complete mandible samples, fully preserved, were selected from the personal collection of the Sabaté family. The sex of these samples was unknown, but all of them belonged to the White Rasquera goat breed. The Blanca de Rasquera goat breed is the only autochthonous goat population located in Catalonia. It is bred for its meat and there are less than 5,000 animals belonging to this breed. All of them were adults (all molars erupted), since skeletal growth was complete, and so, age influences could be discounted. The mandibles were disarticulated and the skulls were not studied. Mandibles were then halved along the median line and both halves were used for the study. None of the mandibles presented apparent pathological deformities or malocclusions.

*Corresponding author

Tel: +34-973-70-64-60, Fax: +34-973-28-74

E-mail: peremiquelp@prodan.udl.cat 

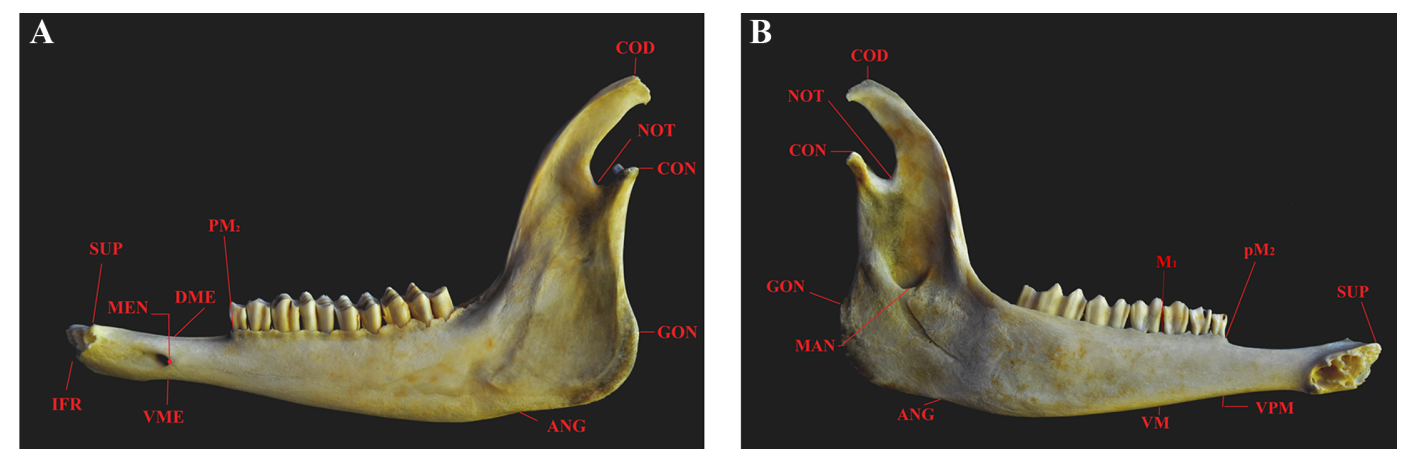

Fig. 1. Lateral (A) and medial (B) planes of the mandible, showing landmarks. COD: Coronion (the highest point of the coronoid process), NOT: deepest point of the mandibular notch, CON: Condylion (the most superior point of the condylar process), SUP: the oral border of the alveoli of the $\mathrm{I}_{4}$, MEN: mental foramen, DME: dorsal point vertical to the mental foramen, $\mathrm{PM}_{2}$ : oral border of the alveolus of $\mathrm{pM}_{2}$, IFR: Infradentale (the most prominent median point at the oral border of the alveoli of the incisors), VME: the base of the mandible at a vertical line from the mental foramen, ANG: angular notch, GON: Gonioncaudale (the most aboral point of the ramus), VM: the base of the mandible at a vertical line from $\mathrm{M}_{1}, \mathrm{VPM}$ the base of the mandible at a vertical line from $\mathrm{pM}_{2}$, MAN: the base of the mandible at a vertical line from the mandibular foramen.

\section{Image capture}

Image capture was performed with a Nikon D70 digital camera (image resolution of 2,240 $\times 1,488$ pixels; Nikon, Japan) equipped with a Nikon AF Nikkor 28-200 mm telephoto lens (Nikon). The focal axis of the camera was parallel to the horizontal plane of reference and centred on the lateral and medial aspects of each hemimandible. A ruler was used in this process (interval $100 \mathrm{~mm}$ ). For the lateral aspect, 11 bilateral landmarks were established (Fig. 1A). For the medial aspect, 10 bilateral landmarks were established (Fig. 1B). The chosen landmarks were referred to as major anatomical mandibular structures. Nomina Anatomica Veterinaria [5] and Driesch [3] were used to guide the spelling of anatomical and zoological terms in this investigation. Two replicas were performed for the lateral aspect. A Mantel test between the two replicates reflected $\mathrm{R}=0.998, p<0.001$, which suggested that the matrix entries were positively associated and thus, digitising error was considered negligible.

The inter-landmark distances were: dorsal point vertical to the mental foramen (DME)-Oral border of the alveolus of $\mathrm{pM}_{2}\left(\mathrm{pM}_{2}\right)$, Infradentale (IFR)-the oral border of the alveoli of the $\mathrm{I}_{4}$ (SUP), angular notch (ANG)- Coronion (COD), ANG- deepest point of the mandibular notch (NOT), ANGCondylion (CON), mental foramen (MEN)-the base of the mandible at a vertical line from the mental foramen (VME), MEN-DME, MEN-SUP, MEN-COD, MEN-NOT, MEN-CON and MEN-ANG (lateral aspect), and SUP-CON, $\mathrm{PM}_{2}-\mathrm{SUP}$, $\mathrm{PM}_{2}$-the base of the mandible at a vertical line from $\mathrm{pM}_{2}$ (VPM), the base of the mandible at a vertical line from the mandibular foramen (MAN)-VPM, MAN-ANG, MAN-GON, MAN-NOT, MAN-CON and MAN-COD (medial aspect). All these measurements allowed a good characterisation of the morphological features in the hemimandible. Distances between landmarks were obtained using the program Traditional Morphometrics Generator (TMorphGen, ver. 6c, Sheets, 2003). All measurements were made by the author.

\section{Mandible size}

The overall size of each hemimandible was evaluated on both sides (right and left) by the distances DME-PM ${ }_{2}$, IFRSUP, ANG-COD, ANG-NOT and ANG-CON for the lateral aspect, and by $\mathrm{PM}_{2}-\mathrm{SUP}$ (diastema) and $\mathrm{PM}_{2}-\mathrm{VPM}$ for the medial aspect. The medial distance SUP-CON was interpreted as the hemimandibular length.

\section{Neural mandible}

The symmetry of the position of the mental (MEN) and mandibular (MAN) foramina was evaluated by seven measurements of the mental foramen (centre), MEN-VME, MENDME, MEN-SUP, MEN-COD, MEN-NOT, MEN-CON and MEN-ANG, and six by the mandibular foramen (inferior border), MAN-VM, MAN-ANG, MAN-GON, MAN-NOT, MANCON and MAN-COD.

\section{Ethics statement}

No specific permits were required for this study as it did not involve the slaughtering of any animal or endangered or protected species.

\section{Data analysis}

For each parameter, the mean and standard deviation (SD) were calculated for both the right and left sides. Paired Student $t$ (Wilcoxon) test and Spearman's rank correlation coefficient $\left(\mathrm{r}_{\mathrm{s}}\right)$ were used to compare data on the left (L) and right (R) sides of each animal using a 5\% statistical significance. For each parameter, the raw unsigned difference $|\mathrm{R}-\mathrm{L}|$ as well as the relative unsigned difference $|\mathrm{R}-\mathrm{L}| /|\mathrm{R}+\mathrm{L}| \times 100$ were computed. This calculation allows the determination of individual differences in size and provides a value for asymmetry of each individual [13]. The result of this ratio-formula gives a range from $0 \%$ (complete symmetry) to $100 \%$ (asymmetry) [13]. It allowed us to compare the relative changes while taking into account the mandibular size. In humans, a 
Table 1. Results for the lateral aspect

\begin{tabular}{|c|c|c|c|c|c|c|c|c|}
\hline Measurement & $\begin{array}{c}\text { Right } \\
\text { Mean } \pm \text { SD }\end{array}$ & $\begin{array}{c}\text { Left } \\
\text { Mean } \pm \text { SD }\end{array}$ & $\begin{array}{r}\text { Paired } \\
t \text { test }\end{array}$ & $\begin{array}{c}p \\
\text { (same mean) }\end{array}$ & $\mathrm{r}_{\mathrm{s}}$ & $p$ & $\begin{array}{l}\text { Raw unsigned } \\
\text { difference }\end{array}$ & $\begin{array}{c}\text { Relative unsigned } \\
\text { difference }(\%)\end{array}$ \\
\hline MEN-VME & $8.50 \pm 1.57$ & $8.37 \pm 1.48$ & 0.49 & 0.63 & 0.75 & 0.00 & 0.83 & 5.02 \\
\hline MEN-DME & $6.07 \pm 0.90$ & $6.37 \pm 0.95$ & 1.49 & 0.16 & 0.54 & 0.04 & 0.62 & 4.98 \\
\hline MEN-SUP & $29.90 \pm 3.41$ & $29.27 \pm 4.26$ & 0.68 & 0.51 & 0.59 & 0.02 & 2.33 & 3.81 \\
\hline${\text { DME}-\mathrm{PM}_{2}}_{2}$ & $19.51 \pm 2.06$ & $19.27 \pm 2.31$ & 0.56 & 0.58 & 0.76 & 0.00 & 1.07 & 2.76 \\
\hline IFR-SUP* & $12.50 \pm 1.67$ & $14.55 \pm 1.37$ & 3.78 & $<0.05$ & 0.07 & 0.81 & 2.25 & 8.39 \\
\hline MEN-COD & $172.04 \pm 7.93$ & $172.44 \pm 7.28$ & 0.58 & 0.57 & 0.90 & 0.00 & 2.22 & 0.65 \\
\hline MEN-NOT & $148.57 \pm 6.79$ & $148.32 \pm 6.10$ & 0.46 & 0.65 & 0.93 & 0.00 & 1.63 & 0.55 \\
\hline MEN-CON & $162.61 \pm 7.26$ & $161.87 \pm 6.59$ & 1.53 & 0.15 & 0.95 & 0.00 & 1.64 & 0.50 \\
\hline MEN-ANG & $150.92 \pm 6.23$ & $150.13 \pm 6.54$ & 1.88 & 0.08 & 0.97 & 0.00 & 1.52 & 0.51 \\
\hline ANG-COD & $108.02 \pm 6.20$ & $107.82 \pm 5.90$ & 0.23 & 0.82 & 0.83 & 0.00 & 2.87 & 1.32 \\
\hline ANG-NOT & $73.31 \pm 4.70$ & $73.04 \pm 4.70$ & 0.37 & 0.71 & 0.70 & 0.00 & 2.33 & 1.59 \\
\hline ANG-CON & $81.19 \pm 5.28$ & $80.76 \pm 4.81$ & 0.55 & 0.59 & 0.76 & 0.00 & 2.73 & 1.67 \\
\hline
\end{tabular}

Means are given in $\mathrm{mm}$. ${ }^{*}$ Significantly different paired measurements appear in the line of IFR-SUP. SD: standard deviation, $\mathrm{r}_{\mathrm{s}}$ : spearman.

Table 2. Results for the medial aspect

\begin{tabular}{|c|c|c|c|c|c|c|c|c|}
\hline Measurement & $\begin{array}{c}\text { Right } \\
\text { Mean } \pm \text { SD }\end{array}$ & $\begin{array}{c}\text { Left } \\
\text { Mean } \pm \text { SD }\end{array}$ & $\begin{array}{r}\text { Paired } \\
t \text { test }\end{array}$ & $\begin{array}{c}p \\
\text { (same mean) }\end{array}$ & $\mathrm{r}_{\mathrm{s}}$ & $P$ & $\begin{array}{l}\text { Raw unsigned } \\
\text { difference }\end{array}$ & $\begin{array}{l}\text { Relative unsigned } \\
\text { difference }(\%)\end{array}$ \\
\hline SUP-CON & $135.42 \pm 5.89$ & $134.75 \pm 5.76$ & 1.14 & 0.28 & 0.94 & 0.00 & 1.77 & 0.66 \\
\hline MAN-VM & $35.91 \pm 3.75$ & $35.67 \pm 3.33$ & 0.73 & 0.48 & 0.93 & 0.00 & 1.05 & 1.46 \\
\hline $\mathrm{PM}_{2}-\mathrm{SUP}$ & $33.00 \pm 1.65$ & $33.84 \pm 2.02$ & 1.73 & 0.10 & 0.80 & 0.00 & 0.89 & 1.30 \\
\hline $\mathrm{PM}_{2}-\mathrm{VPM}$ & $14.88 \pm 1.13$ & $14.68 \pm 1.16$ & 0.71 & 0.49 & 0.53 & 0.04 & 0.72 & 2.50 \\
\hline MAN-ANG & $53.62 \pm 4.31$ & $52.67 \pm 5.25$ & 0.98 & 0.34 & 0.63 & 0.01 & 2.82 & 2.64 \\
\hline MAN-GON & $47.50 \pm 4.47$ & $47.70 \pm 6.12$ & 0.14 & 0.89 & 0.49 & 0.07 & 4.28 & 4.44 \\
\hline MAN-NOT & $14.84 \pm 1.96$ & $15.46 \pm 1.88$ & 0.85 & 0.41 & 0.04 & 0.89 & 2.06 & 6.96 \\
\hline MAN-CON & $72.91 \pm 4.24$ & $73.42 \pm 5.50$ & 0.71 & 0.49 & 0.75 & 0.00 & 1.99 & 1.37 \\
\hline MAN-COD & $14.84 \pm 1.96$ & $15.46 \pm 1.88$ & 0.85 & 0.41 & 0.04 & 0.89 & 2.06 & 6.96 \\
\hline
\end{tabular}

Means are given in $\mathrm{mm}$.

relative unsigned difference superior to $3 \%$ is considered clinically discernible [7]. In the case of symmetry, the modal distribution of signed right-left differences was assessed using the Shapiro-Wilk test using 5\% statistical significance.

Data were analysed using PAST (Paleontological Statistics Software Package for Education and Data Analysis) [4].

\section{Results}

\section{Mandible size}

The total length of the hemimandible (distance SUP-CON) was equal on both sides $(\mathrm{t}=1.14, p<0.05)$ : $135.42 \pm 5.89$ and $134.75 \pm 5.76 \mathrm{~mm}$ for the right and left hemimandibles, respectively, which were also well correlated $(p<<0.001)$. The mean linear difference for the mandible size ranged from 0.50 (MEN-CON) to 8.39 (IFR-SUP).

On the lateral aspect (Table 1), there was only a significant asymmetry in the IFR-SUP distance, which was clearly large in the left hemimandible. The relative unsigned difference between the sides was high $(8.4 \%)$, but the variation was symmetrically distributed around 0 (Shapiro-Wilk's W= $0.912, p=0.149$ ). On the medial aspect (Table 2), there was only a significant asymmetry in the $\mathrm{PM}_{2}$-SUP distance, which was slightly larger at the left side. Although the right diastema tended to be shorter, the relative unsigned difference between the sides was low $(1.3 \%)$ and the distribution was unimodal with a mean of zero and a variation symmetrically distributed around 0 (Shapiro-Wilk's $\mathrm{W}=0.934, p=0.314$ ).

\section{Neural mandible}

The positions of the mental and mandibular foramina were symmetric for the studied samples. The relative differences superior to 3\% corresponded to distances linked to MEN and MAN, except for the IFR-SUP distance. Relative unsigned differences tended to be larger for MEN and MAN, which were also positively related to mandible size. 


\section{Discussion}

Although the mandible is described in humans as an asymmetric bone $[9,10,12]$, only the diastema differed significantly between the sides in our studied goat sample. Moreover, this was related to the rostral part (incisive arch). No side dominance appeared. The action of the functional matrix (masticatory muscles and tongue, among others) is essential for bone formation. The observed differences in the amount of deviation of the symmetries, although higher than $3 \%$ for some distances, were not evident upon visual inspection of the studied mandibles and did not alter masticatory function (an altered masticatory function leading to a significant difference in the amount of deviation in the mandible would cause the animal to be discarded for breeding purposes). Thus, the value of $3 \%$ as a clinical reference to calculate symmetry in human mandible $[8,2]$ seems not to be applicable to domestic goats. If unsigned asymmetry is interpreted as a measure of developmental stability, then the studied breed presents a marked ability to develop in good fitness despites its harsh environment. According to ontogeny, the mandible is a structure composed of several subunits, which are derived from distinct developmental origins and assemble to form an integrated whole. If the incisive region is the least symmetrical region of the caprine mandible, this indicates that this modular structure is more conservative for the neural part.

Much remains to be done, however, to understand how the many processes involved in forming the mandible interact to join its components together into a coherent functional unit.

\section{Acknowledgments}

Very special thanks are extended to the farmers who facilitated initial sample collection from their herds, and specially to Josué Sabaté, who sampled all the specimens. The author would like to thank the two anonymous reviewers for their valuable comments and suggestions to improve the manuscript.

\section{References}

1. Barone R. Anatomie Comparée des mammifères domestiques. pp. 761, Vigot, Paris, 1999.

2. Captier G, Lethuilier J, Oussaid M, Canovas F, Bonnel F. Neural symmetry and funciontal asymmetry of the mandible. Surg Radiol Anat 2006, 28, 379-386.

3. von den Driesch A. A Guide to the Measurement of Animal Bones from Archaeological Sites. Bulletin 1. pp. 1137, Peabody Museum of Archaeology and Ethnology, Harvard University, Cambridge, 1976.

4. Hammer Ø, Harper DAT, Ryan PD. Past: paleontological statistics software package for education and data analysis. Palaeontol Electronica 2001, 4, 4.

5. ICVGAN. Nomina Anatomica Veterinaria. 5th ed. World Association of Veterinary Anatomists, Knoxville, 2005

6. König HE, Liebich HG. Veterinary Anatomy of Domestic Mammals: Textbook and Colour Atlas. pp. 768, Schattauer, Sttutgart, 2009.

7. Lu KH. Harmonic analysis of the human face. Biometrics 1965, 21, 491-505.

8. Luz JGC, Miyazaki LT, Rodrigues L. Verification of the symmetry of the mandibular ramus in patients with temporomandibular disorders and asymptomatic individuals: a comparative study. Bull Group Int Rech Sci Stomatol Odontol 2002, 44, 83-87.

9. Pierrakou ED. Asymmetry of the mandible. Study of 386 mandibles in vitro. Hell Period Stomat Gnathopathoprosopike Cheir 1990, 5, 13-16.

10. Ponyi S, Szabó G, Nyilasi J. Asymmetry of mandibular dimensions in European skulls. Proc Finn Dent Soc 1991, 87, 321-327.

11. Sperber GH. Craniofacial Development. pp. 220, B.C. Decker, London, 2001.

12. Türp JC, Alt KW, Vach W, Harbich K. Mandibular condyles and rami are asymmetric structures. Cranio 1998, 16, 51-56.

13. Uthman AT, Al-Rawi NH. Orthopantomographic assessment of mandibular asymmetry as an aid in diagnosis of temporomandibular problems. J Bagh Coll Dentistry 2006, 18, 40-42. 